\title{
Deriving consistent pairwise comparison matrices in decision making methodologies based on linear programming method
}

\author{
Haiqing Zhang*, Aicha Sekhari", Yacine Ouzrout ${ }^{*}$, and Abdelaziz Bouras ${ }^{* *}$ \\ *DISP laboratory, University Lumière Lyon 2, France, 160 Bd de l'Université 69676 Bron Cedex \\ †Computer Science Dept., Faculty of Engineering, Qatar University, Box. 2731, Doha, Qatar \\ \{aicha.sekhari,yacine.ouzrout,xi.yu\}@univ-lyon2.fr \\ abdelaziz.bouras@qu.edu.qa
}

\begin{abstract}
Pairwise comparison matrix (PCM) with crisp or fuzzy elements should satisfy consistency requirements when it is used in analytic hierarchy process (AHP) or in fuzzy AHP methodologies. An algorithm has been presented to obtain a new modified consistent PCM for the corresponding inconsistent original one. The algorithm sets a linear programming problem based on all of the constraints. To obtain the optimum eigenvector of the middle value of the new PCM, segment tree is used to gradually approach the greatest lower bound of distance with the original PCM. As to obtain the lower value and upper value of the new PCM, a theory is proposed to reduce adding uncertainty factors and could maximum maintain the similarity with original PCM. The experiments for crisp elements show that the proposed approach can preserve more the original information than references. The experiments for fuzzy elements show that our method can effectively reduce inconsistency and obtain suitable modified fuzzy PCMs.
\end{abstract}

Keywords: AHP, Fuzzy AHP, Inconsistency pairwise comparison matrix, Preserve information, Decision Making

\section{Introduction}

Analytic Hierarchy Process (AHP) [1, 10-11] and Fuzzy AHP [12-13] are multi criteria decisionmaking methodologies which are widely used in many real problems. The AHP expresses the relative importance of criteria by pairwise comparisons and converts the values of pairwise comparisons to priorities. However, AHP method has the inability to handle uncertain and imprecise situations due to the difficulty of mapping decision-makers' preferences into crisp value [34]. Fuzzy sets theory can appropriately handle uncertainty and imprecision issues [31, 7, and 8]. It can better describe vague demands or capacities with tolerance than probability distributions. It is also a sophisticated technique to solve real problems when data is subject to imprecision. Fuzzy AHP methodology [12-13] adopts triangular (or trapezoidal) membership functions to define fuzzy sets of decision-makers' preferences. It derives the fuzzy priorities of criteria from pairwise comparisons matrix with triangular (or trapezoidal) fuzzy elements to tackle the uncertainty and inaccurate issues in multicriteria decision-making process. To make sure the priorities of each criterion are accurate and sensible in either AHP or Fuzzy AHP, the consistency of Pairwise Comparison Matrix (PCM) with crisp or fuzzy elements must be achieved. For the PCMs which fail the consistency test, the decision-makers must redo the ratios. To be able to facile employ AHP or Fuzzy AHP, a strategy should be proposed to repair the inconsistency information of PCMs.

Some works $[3-5,35]$ focus on studying inconsistency issues with crisp numbers. Karapetrovic and Rosenbloom [3] revise the single entry of a ratio's value till consistency of relative PCM (pairwise comparison matrix) matrix in an acceptable level. Xu and Wei [4] preserve the initial ratios' value in PCM 
(pairwise comparison matrix) while obtaining satisfied consistency requirements. Cao et al. [5] develop a heuristic approach which can preserve more original comparison information compared with $\mathrm{Xu}$ and Wei [4]. Chiclana et al. [35] present functional equation to evaluate cardinal consistency of reciprocal preference relations and provide results toward the construction of consistent reciprocal preference relations. However, for these three works, the computing times are largely increased and the information of original matrix cannot be well preserved when the consistency requirement is increased. To find out an approximate PCM which is consistent and closest to the optimal one, we need to minimize the distance between inconsistent PCMs and their corresponding consistent PCMs.

Some works [6, 18, 25, and 36] are used to solve the inconsistency issues with fuzzy elements. $\mathrm{Xu}$ and Wang [6] repaire incomplete inconsistency fuzzy preference relation by finding out the unusual and false element until the consistency ratio was at a satisfied level. Leung and Cao [18] propose a new definition of Fuzzy positive reciprocal matrix by setting deviation tolerances based on an idea of allowing inconsistent information. Zadeh and Bafandeh [25] further discuss Leung and Cao's work and proposed a new method of fuzzy consistency test by direct fuzzification of QR (Quick Response) algorithm which is one of the methods for the eigenvalues calculating of an arbitrary matrix. Cabrerizo et al. [36] adopt consistency measures in an iterative procedure to estimate the incomplete information, and the proposed consensus model supports the management of incomplete unbalanced fuzzy linguistic information. However, these works do not have standard parameters to criticize the reliability of their theory so far. Therefore, it is very important to prove the feasibility of a methodology that can reduce the inconsistency of the original matrix and preserve the original matrix's information as much as possible.

An algorithm is proposed to obtain the new modified PCM based on eigenvector method and linear programming method. The modified PCM is considered as a combination of the original inconsistent PCM and an adjustable consistent PCM. The algorithm aims to obtain the middle value, upper value, and lower value of adjustable matrix. The strategy is to find the optimum priority vector by solving a linear programming problem, and use eigenvector method to obtain the adjustable matrix based on the optimum priority vector. This paper is structured in the following way. Section 2 gives the basic concepts of the PCM with fuzzy and crisp elements, consistency indices, and parameters to judge the effectiveness of modified matrix. Section 3 gives the detail of the proposed algorithm. Section 4 and section 5 apply the algorithm to obtain the modified PCMs with crisp and fuzzy elements. Section 6 concludes our work.

\section{Preliminaries}

\subsection{Pairwise Comparison matrixes with triangular fuzzy elements}

Triangular fuzzy numbers are special cases of trapezoidal fuzzy numbers, which are widely used in fuzzy applications [12]. Triangular fuzzy number allows decision-makers to provide preferences in a fuzzy range that can keep certain unknown ratio [19]. Our work studies show how to obtain a consistent pairwise comparison matrix with triangular fuzzy elements when given an inconsistent one.

The $m \times n$ pairwise comparison matrix Ã with triangular fuzzy elements can be described in the following:

$$
\tilde{\mathrm{A}}=\left(\begin{array}{ccc}
\left(a_{11}^{L}, a_{11}^{M}, a_{11}^{U}\right) & \ldots & \left(a_{1 n}^{L}, a_{1 n}^{M}, a_{1 n}^{U}\right) \\
\vdots & \ddots & \vdots \\
\left(a_{m 1}^{L}, a_{m 1}^{M}, a_{m 1}^{U}\right) & \cdots & \left(a_{m n}^{L}, a_{m n}^{M}, a_{m n}^{U}\right)
\end{array}\right)
$$

Where for each element $\tilde{a}_{i j}=\left(a_{i j}^{L}, a_{i j}^{M}, a_{i j}^{U}\right), a_{i j}^{L}$ is the lower value, $a_{i j}^{M}$ is the middle value, and $a_{i j}^{U}$ is the upper value. In the particular situation, if $\mathrm{m}=\mathrm{n}$, and the following condition is satisfied:

$\tilde{a}_{i j}=\left(a_{i j}^{L}, a_{i j}^{M}, a_{i j}^{U}\right)$ implies $\tilde{a}_{j i}=\left(\frac{1}{a_{i j}^{U}}, \frac{1}{a_{i j}^{M}}, \frac{1}{a_{i j}^{L}}\right),(\forall \mathrm{i}$, $\mathrm{j}=1,2, \ldots, \mathrm{n})$, then, $\tilde{\mathrm{A}}$ is a reciprocal pairwise comparison matrix. Where,

$1 / \sigma<a_{i j}^{L}<a_{i j}^{M}<a_{i j}^{U}<\sigma$ and $S=[1 / \sigma, \sigma], \sigma>1$. S is an interval of real numbers called fuzzy scale. Crisp numbers (non-fuzzy numbers) are special cases of $\tilde{\mathrm{A}}$ when $a_{i j}^{L}=a_{i j}^{M}=a_{i j}^{U}$.

Definition 1. It was proposed in Buckley [2], a fuzzy positive reciprocal matrix $\tilde{\mathrm{A}}=\left[\widetilde{a_{i j}}\right]_{n \times n}$ is consistent if and only if $\tilde{a}_{i k} \odot \tilde{a}_{k j} \approx \tilde{a}_{i j},\{\forall \mathrm{i}, \mathrm{j}, \mathrm{k} \mid 1 \leq \mathrm{i}, \mathrm{j}, \mathrm{k}$ $\leq \mathrm{n}\}$.

Where, the operator $\odot$ is one of the operation rules of triangular fuzzy elements. This operation can be calculated by the following equation: 


$$
\begin{aligned}
\tilde{a}_{i k} \odot \tilde{a}_{k j} & =\left(a_{i k}^{L}, a_{i k}^{M}, a_{i k}^{U}\right) \odot\left(a_{k j}^{L}, a_{k j}^{M}, a_{k j}^{U}\right) \\
& =\left(a_{i k}^{L} \bullet a_{k j}^{L}, a_{i k}^{M} \bullet a_{k j}^{M}, a_{i k}^{U} \bullet a_{k j}^{U}\right) \approx \underset{\tilde{\mathrm{A}}}{\tilde{a_{i j}}}=\left(a_{i j}^{L}, a_{i j}^{M}, a_{i j}^{U}\right)
\end{aligned}
$$

When positive reciprocal matrix $\tilde{\mathrm{A}}_{\text {is crisp numbers }}$ $\mathrm{A}$, consistent condition is $a_{i k} \bullet a_{k j}=a_{i j},\{\forall \mathrm{i}, \mathrm{j}, \mathrm{k} \mid 1 \leq \mathrm{i}$, $\mathrm{j}, \mathrm{k} \leq \mathrm{n}\}$.

However, this definition is too strict because it is unrealistic to reach perfect consistency of a PCM (crisp or fuzzy elements). Some works [14-21] have developed consistency indices to accept a certain level of acceptable deviations. We will study consistency indices to decide whether the current PCM should be in an acceptable consistency level or not.

\subsection{Consistency Indices for pairwise comparison matrix}

Several consistency indices have been proposed for crisp numbers. For example: Geometric Consistency Index [14], singular value decomposition method [15], harmonic consistency index [16], etc. A large majority of indices have a good internal agreement, whereas few indices are outliers which have weaker agreement when compared with the other indices. Different consistency indices are different definition of consistency degree and there is still no evidence to show that which proposal is the best one. We use the most popular and oldest index of Satty'CR [17] to measure the consistency of crisp numbers.

The consistency index of CR [17] is defined as:

$$
C R=\left(\left(\lambda_{\max }-n\right) /(n-1)\right) \cdot(1 / R I)
$$

Where $\lambda_{\max }$ is the principle eigenvalue of A. RI is random index which can be gotten by searching defined table. When the value of $0<\mathrm{CR}<0.1$, the consistency can be accepted.

Several important works focus on the consistency of pairwise comparison matrix with fuzzy elements. The first one is Leung and Cao [18], who proposed a notion with consideration of a tolerance deviation. However, the notion is strongly related to Satty's $C R$ and it has shortcomings to calculate consistency of pairwise comparison matrix with fuzzy elements [25]. The second one is Ramik and Jaroslav [19]'s work, which proposes a new consistency index NI to examine fuzzy elements based on the distance of the matrix to a special ratio matrix, and compare the properties with $C R$. This work has been further studied and used by several important works [14, 20-21]. We have tested this work's performance which indicated that it can satisfy reasonable results with fuzzy elements, although it has some shortcomings [21].

The consistency index of NI [19] is defined as:

$$
\begin{aligned}
& N I_{n}^{\sigma}=\gamma_{n}^{\sigma} \cdot \max _{i, j}\left\{\operatorname { m a x } \left\{\left|\left(\omega_{i}^{L} / \omega_{j}^{U}\right)-a_{i j}^{L}\right|,\right.\right. \\
& \left.\left.\quad\left(\omega_{i}^{M} / \omega_{j}^{M}\right)-a_{i j}^{M}|,|\left(\omega_{i}^{U} / \omega_{j}^{L}\right)-a_{i j}^{U} \mid\right\}\right\},(1 \leq i, j \leq n)
\end{aligned}
$$

Where, $\gamma_{n}^{\sigma}$ is a normalization factor, the value of $\omega_{i}^{L}, \omega_{i}^{M}, \omega_{i}^{U}$ can be obtained from $a_{i j}^{L}, a_{i j}^{M}, a_{i j}^{U}$.

The third and the forth work are [27, 28]'s study, they extend GCI (Geometric Consistency Index) [26] to CCI (centric consistency index) index to deal with PCM with triangle fuzzy elements. The consistency index CCI is defined as:

$$
\begin{aligned}
C C I(\mathrm{~A}) & =(2 /(n-1)(n-2)) \\
& \times \sum_{i<j}\left(\log \left(\left(a_{i j}^{L}+a_{i j}^{M}+a_{i j}^{U}\right) / 3\right)\right. \\
& \left.\log \left(\omega_{i}^{L}+\omega_{i}^{M}+\omega_{i}^{U} / \omega_{j}^{L}+\omega_{j}^{M}+\omega_{j}^{U}\right)\right)^{2}
\end{aligned}
$$

Where $\mathrm{A}=\left(a_{i j}^{L}, a_{i j}^{M}, a_{i j}^{U}\right)$ are the elements for fuzzy PCM, $\omega=\left(\omega_{i}\right)$ is priority vector derived by logarithmic least squares. When $\operatorname{CCI}(\mathrm{A})=0$, $\mathrm{A}$ is considered as fully consistent. Thresholds remain identical with index GCI as CCI is a fuzzy extension of GCI. The thresholds are provided as: $\mathrm{CCI}=0.3147$ for $\mathrm{n}=3, \mathrm{CCI}=0.3526$ for $\mathrm{n}=4$ and $\mathrm{CCI}=0.370$ for $\mathrm{n}>4$ based on Aguaron's [26].

\subsection{Parameters to judge the effectiveness of modified matrix}

Fewer works describe the necessary parameters which can be used to measure the effectiveness of modified PCM B. Xu and Wei [4] have given two parameters in the following:

$$
\begin{aligned}
& \delta=\max _{i, j}\left\{\left|b_{i j}-a_{i j}\right|\right\}, i, j=1,2, \cdots, n \\
& \sigma=\sqrt{\sum_{i=1}^{n} \sum_{j=1}^{n}\left(b_{i j}-a_{i j}\right)^{2}} / n
\end{aligned}
$$

$\boldsymbol{\sigma}$ and $\boldsymbol{\delta}$ are used as the parameters of modificatory effectiveness. The authors $[4,5]$ argue that the modified matrix that preserves the most information of the original one must satisfy the condition: $0<\delta<2 \& 0<\sigma<1$. We extend these two parameters to be suitable to judge modificatory effectiveness of fuzzy elements, and the range is identical with $\sigma$ and $\delta . \sigma_{f u z z}$ and $\delta_{f u z z y}$ are: 


$$
\begin{aligned}
& \delta_{\text {fuzzy }}=\max _{i, j}\left\{\left|b_{i j}-\widetilde{a}_{i j}\right|\right\}=\max _{i, j}\left\{\left|b_{i j}^{L}-a_{i j}^{L}\right|,\left|b_{i j}^{M}-a_{i j}^{M}\right|,\left|b_{i j}^{U}-a_{i j}^{U}\right|\right\}, i, j=1,2, \cdots, n \\
& \sigma_{\text {fuzzy }}=\left(\sqrt{\left.\max \left\{\left(\sum_{i=1}^{n} \sum_{j=1}^{n}\left(b_{i j}^{L}-a_{i j}^{L}\right)^{2}\right),\left(\sum_{i=1}^{n} \sum_{j=1}^{n}\left(b_{i j}^{M}-a_{i j}^{M}\right)^{2}\right),\left(\sum_{i=1}^{n} \sum_{j=1}^{n}\left(b_{i j}^{U}-a_{i j}^{U}\right)^{2}\right)\right\}\right)} / n\right.
\end{aligned}
$$

Besides $\sigma$ and $\delta$, two parameters proposed by $\mathrm{Xu}$ and Wei [4], a third parameter should be added, which is Condition of Order Preservation (COP) [24]. For example, suppose the original matrix A has alternatives $\left(a_{1}, a_{2}, a_{3}, a_{4}, a_{5}, a_{6}, a_{7}, a_{8}\right)$, it has the relationship $a_{1}$ dominates $a_{2}$, $a_{3}$ dominates $a_{4}$, and the judgments indicate that the extent to which $\mathrm{a}_{1}$ dominates $a_{2}$ is greater that the extent to which $a_{3}$ dominates $a_{4}$, then the priority vector $\omega$ should satisfy: $\omega\left(\mathrm{a}_{1}\right)>\omega\left(\mathrm{a}_{2}\right)$ and $\omega\left(\mathrm{a}_{3}\right)>\omega\left(\mathrm{a}_{4}\right)$ (preservation of order of preference), and $\omega\left(\mathrm{a}_{1}\right) / \omega\left(\mathrm{a}_{2}\right)>\omega\left(\mathrm{a}_{3}\right) / \omega\left(\mathrm{a}_{4}\right)$ (preservation of order of intensity of preference).

\section{Deriving the optimum eigenvector by solving linear programming problem to obtain modified pairwise comparison matrix}

Three conditions can guarantee a new modified consistent PCM B(or B) which has the closest and maximal similarity with the orginal inconsistent PCM A (or A):

1). The consistent value should be at an acceptable level based on different consistency indices;

2). The farthest distance between new PCM $\mathrm{B}$ (or B) and orginal PCM A (or A) should be as small as possible;

3). The obtained new matrix should have a strong similarity with the orginal matrix.

To suit these conditions, we provide an adjustable PCM which can reduce the inconsistency of orginal PCM as much as possible. On the basis of the adjustable matrix, we propose the definition of the modified matrix

Definition 2. The new modified PCM B is defined as a combination of the original PCM A and an

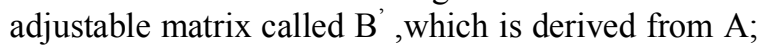
the modified PCM B is defined in equation (3.1):

$$
B=a_{i j}{ }^{\beta}\left(b_{i j}{ }^{1-\beta}\right.
$$

For fuzzy elements, the new modified PCM Bis defined as:

$$
\begin{aligned}
B= & \left\{\left(\left(a_{i j}^{L}\right)^{\beta} \cdot\left(b_{i j}^{L^{\prime}}\right)^{1-\beta}\right),\left(\left(a_{i j}^{M}\right)^{\beta} \cdot\left(b_{i j}^{M^{\prime}}\right)^{1-\beta}\right),\right. \\
& \left.\left(\left(a_{i j}^{U}\right)^{\beta} \cdot\left(b_{i j}^{U^{\prime}}\right)^{1-\beta}\right)\right\}
\end{aligned}
$$

The new modified matrix is constructed by two parts based on definition 2: 1). One is the original matrix. The function of this matrix is to keep the original information and make sure the two matrixes is in an acceptable distance. 2). One is the adjustable matrix. The function of this matrix is to modify the inconsistency level to make sure the new modified matrix's consistency is based on consistency indexes.

One way to be able to obtain the adjustable PCM is by one element being $B^{\prime}$ is $b_{i j}{ }^{\prime}=\left(\left(b_{i j}^{L}\right)^{\prime},\left(b_{i j}^{M}\right)^{\prime},\left(b_{i j}^{U}\right)^{\prime}\right)$ and one element of $\tilde{\mathrm{A}}$ is $\tilde{a}_{i j}=\left(a_{i j}^{L}, a_{i j}^{M}, a_{i j}^{U}\right)$, by making every element of $B^{\prime}$ closely resemble the elements of $\tilde{A}$, which is $\left(b_{i j}^{L}\right)^{\prime} \doteq a_{i j}^{L},\left(b_{i j}^{M}\right)^{\prime} \doteq a_{i j}^{M}$, and $\left(b_{i j}^{U}\right)^{\prime} \doteq a_{i j}^{U}$. Therefore, we go to find $\left(b_{i j}^{L}\right)^{\prime},\left(b_{i j}^{M}\right)^{\prime},\left(b_{i j}^{U}\right)^{\prime}$ separately. After finding out $\left(b_{i j}^{L}\right)^{\prime},\left(b_{i j}^{M}\right)^{\prime},\left(b_{i j}^{U}\right)^{\prime}$, combine them together to get the new matrix $B^{\prime}$.

\subsection{Deriving the middle value of the adjustable matrix}

\subsubsection{Distance analysis between original matrix and adjustable matrix}

The distance methods have logarithmic least square method (LSM)[29], eigenvector method $[23,24]$, and least squares method [30]. The optimum eigenvector should be as close as possible to the original eigenvector (derived from the original matrix). The adjustable matrix should strongly resemble the original matrix once the optimum eigenvector has been determined. Therefore, eigenvector can be used to calculate adjustable PCMs.

According to references [22, 23], if matrix $\mathrm{A}$ is consistent, then we could find positive weights $\omega=$ $\left\{\omega_{1}, \omega_{2}, \ldots, \omega_{\mathrm{n}}\right\}^{\mathrm{T}}$ which can satisfy such condition [9, 22, 23]: $a_{i j}=\omega_{i} / \omega_{j}, \forall i, j \in(1,2, \ldots, n)$. Therefore, if matrix $\mathrm{A}$ is close to consistent, then it must have $\left(\omega_{i} / \omega_{j}\right)-a_{i j} \approx 0$. The obtained positive weights $\omega$ can be used to define the expected adjustable PCM. On the basis of this idea, we want to make the fastest distance between $\omega_{i} / \omega_{j}$ and $a_{i j}$ are the minimum value. The fastest distance between $\omega_{i} / \omega_{j}$ and $a_{i j}$ is 
defined as:

$$
f=\max \left(\left|a_{i j}-\omega_{i} / \omega_{j}\right|\right)
$$

The issue of finding the optimal adjustable PCM with crisp elements can be portrayed as resolving the minimum value of function $f$, which can be expressed in equation (3.4):

$$
G(A, \omega)=\min (f)=\min \left(\max \left(\left|a_{i j}-\omega_{i} / \omega_{j}\right|\right)\right)
$$

This equation (3.4) is extended into equation (3.5) to solve adjustable PCM with fuzzy elements:

$$
\begin{aligned}
G(\mathrm{~A}, \omega)= & \min \left(\operatorname { m a x } \left(\left|a_{i j}^{L}-\omega_{i}^{L} / \omega_{j}^{L}\right|,\left|a_{i j}^{M}-\omega_{i}^{M} / \omega_{j}^{M}\right|,\right.\right. \\
& \left.\left.\left|a_{i j}^{U}-\omega_{i}^{U} / \omega_{j}^{U}\right|\right)\right)
\end{aligned}
$$

Eq.(3.4) and Eq.(3.5) can reach the absolute minimum value (lowest point) when even the worst situation of proportion of $\omega_{i} / \omega_{j}$ is closest to $a_{i j}$. The idea to obtain the optimum positive eigenvector $\omega(\omega)$ is to find out all possible constraints and solve feasible solutions by selecting a suitable linear programming pattern in section 3.1.2.

\subsubsection{Build Linear Programming Problem by defined inequalities of the middle value of the adjustable matrix}

In this section, we aim to find out the least absolute worst distance by setting a more precise priority weights range and adding slack variables. The least absolute worst distance is used to achieve the middle value of the adjustable PCM. The middle value of $b_{i j}^{M}$, from adjustable matrix $B^{\prime}$ should have a strong relationship with $a_{i j}^{M} \cdot \tilde{\omega}^{M}$ is the optimum eigenvector matrix of $a_{i j}^{M} \cdot b_{i j}^{M}$, can be gotten in the following equation:

$$
b_{i j}^{M \prime} \approx \omega_{i}^{M} / \omega_{j}^{M}
$$

The optimum value can be gotten when the worst proportion is closest to $a_{i j}^{M}$. Eq.(3.6) has feasible solutions which means the following equation reaches to the minimum based value on Eq.(3.5):

$$
G\left(a_{i j}^{M}, \omega^{M}\right)=\min \left(\max \left(\left|a_{i j}^{M}-\omega_{i}^{M} / \omega_{j}^{M}\right|\right)\right)
$$

We simply write the variable by introducing an additional variable $\mathrm{Z}=G\left(a_{i j}^{M}, \omega^{M}\right)$. Then, the problem is: $\min Z$

$$
\begin{aligned}
& \text { s.t. } \\
& \left|a_{i j}^{M}-\omega_{i}^{M} / \omega_{j}^{M}\right| \leq Z, \forall i, j \in(1,2, \ldots, n) \\
& 1 / \sigma \leq \omega_{i}^{M}, \omega_{j}^{M} \leq \sigma, j=1,2, \ldots, n \text { (3.9) }
\end{aligned}
$$

Assume the value of $\mathrm{Z}$ is given, and then the constraint (3.8) can be rewritten as:

$$
\begin{aligned}
& -Z \leq\left(a_{i j}^{M}-\omega_{i}^{M} / \omega_{j}^{M}\right) \leq Z \\
& \left(a_{i j}^{M}-Z\right) \bullet \omega_{j}^{M}-\omega_{i}^{M} \leq 0 \\
& \omega_{i}^{M} \leq\left(a_{i j}^{M}+Z\right) \bullet \omega_{j}^{M}
\end{aligned}
$$

Take the reciprocal of constraint (3.12); then the new constraint (3.13)-(3.15) and can be gotten.

$$
\begin{aligned}
& \omega_{j}^{M} / \omega_{i}^{M} \geq 1 /\left(a_{i j}^{M}+Z\right) \\
& \omega_{i}^{M} /\left(a_{i j}^{M}+Z\right)-\omega_{j}^{M} \leq 0 \\
& \omega_{j}^{M} /\left(a_{j i}^{M}+Z\right)-\omega_{i}^{M} \leq 0
\end{aligned}
$$

Combine constraint (3.11) and constraint (3.15) and remove one of the inequalities, and then the new inequality can be gotten:

$$
-\omega_{i}^{M}+\left(\max \left\{\left(a_{i j}^{M}-Z\right), \frac{1}{\left(a_{j i}^{M}+Z\right)}\right\}\right) \omega_{j}^{M} \leq 0
$$

Analogously, a similar inequality can be gotten:

$$
-\omega_{j}^{M}+\left(\max \left\{\left(a_{j i}^{M}-Z\right), \frac{1}{\left(a_{i j}^{M}+Z\right)}\right\}\right) \omega_{i}^{M} \leq 0
$$

Next, we add slack variable $S, T$, and objective function $H=S+T$, to change the constraints (3.16) and (3.17) to equality constraints (3.18) and (3.19). Then we specify constraints, propose formulas to calculate $\omega_{i}{ }^{M}, \omega_{j}{ }^{M}$, and add additional stopping parameter $\mathrm{X}$. Then constraints correspond to the following linear programming problem:

$\min X$

s.t.

$$
\begin{aligned}
& -\omega_{i}{ }^{M}+\left(\max \left\{\left(a_{i j}{ }^{M}-Z\right), \frac{1}{\left(a_{j i}{ }^{M}+Z\right)}\right\}\right) \omega_{j}{ }^{M}+S=0 \\
& -\omega_{j}{ }^{M}+\left(\max \left\{\left(a_{j i}{ }^{M}-Z\right), \frac{1}{\left(a_{i j}{ }^{M}+Z\right)}\right\}\right) \omega_{i}^{M}+T=0
\end{aligned}
$$

$\mathrm{H}=S+T$

$\omega_{1}^{M}+X=1$

$1 / \sigma \leq \omega_{i}{ }^{M}, \omega_{j}{ }^{M} \leq \sigma i, j=1,2, \ldots, n$

$0 \leq Z, S, T, H$ 


\section{Remark 1.}

1). In constraint (3.18), we define $\omega_{1}^{M}=1$ to normalize vector $\omega^{M}$. If the stopping parameter $\mathrm{X}=0$, then it means constraint (3.18) has a solution, and the problem (3.18)-(3.23) has a feasible solution; the values of $\omega_{i}{ }^{M}$ is the optimal solution. If the stopping parameter is $\mathrm{X}=1$ which contradicts with constraint (3.21), then the problem (3.18)-(3.23) is inconsistent; then the values of $\omega_{i}^{M}$ is not the optimal solution.

2). The equalities (3.18)-(3.23) can be solved by Simplex algorithm [32]. The main idea of this algorithm is to walk along edges of the polytope to find out extreme points with lower and lower objective values till the minimum value is reached or an unbounded edge is visited. If the extreme point is reached, then the problem (3.18)-(3.23) have feasible solutions.

3). If $Z=Z^{\prime}$ can make problem (3.18)-(3.23) have a feasible solution, then it must have $Z \geq Z$ ' that can also make the problem (3.18)-(3.23) have a feasible solution. The linear programming problem has the optimum solution when inequalities ((3.18)-(3.23)) reach to the greatest lower bound of $\mathrm{Z}$.

4). If $\mathrm{i}=\mathrm{j}$, then $a_{i i}{ }^{M}=1$, then constraint (3.18) can be always be satisfied. Analogous, constraint (3.20) is always satisfied. Then the equalities (3.18)-(3.23) can be used to find adjustable PCM B' for original PCM A.

\subsubsection{Find feasible solutions to solve the linear programming problem}

The aim of this section is to find the feasible solutions of ((3.18)-(3.23)) and determine the greatest lower bound of $Z$. The problem can be described as storing intervals of $\left[0, Z_{\max }\right]$, analyzing the corresponding $X$ value, and finding the greatest lower of $Z$ that makes $X=0$, which can be solved by segment tree. The segment tree is special for storing intervals. The built time is $\mathrm{O}(\mathrm{n} \log \mathrm{n})$ for $\mathrm{n}$ intervals, and it uses $\mathrm{O}(\mathrm{n} \log \mathrm{n})$ storage. The reason we adapted to the segment tree is because: the segments can be stored in any arbitrary manner, it can easily be adapted to counting queries, and it helps us to query the number of segments that contain a given point. The steps to solve inequalities ((3.18)-(3.23)) are as follows:

Step 1 sets the initial value. Assume the accuracy level is $\xi$; let the initial value of $\omega_{i}^{M}$ be $\left(\prod_{j=1}^{n} a_{i j}^{M}\right)^{1 / n}$, and $\omega_{1}{ }^{M}=1$; let $Z_{\max }=\mathrm{Z}=G\left(a_{i j}^{M}, \omega^{M}\right)$, and $Z_{\min }=\mathrm{Z}=0$.
Step 2 builds a segment tree by using interval [0, $\mathrm{Z}$ ]. Let P1, P2, P3, P4 be the list of distinct interval endpoints. We separate intervals into two parts in every division and terminate this process till the value of the interval is less than the accuracy level $(\xi)$. Then obtain the $X$ value in problem ((3.18)-(3.23)) by setting the current $Z$ value. If $X=0$, then the next $Z$ value is equal to the lower bound of the current node. The calculation steps will end till it reaches to endpoints (P1; P2; P3; P4) based on accuracy level $\xi$.

Step 3 selects the greatest lower value of $\mathrm{Z}$ when $\mathrm{X}=0$, inputs $\mathrm{Z}$ value to inequalities ((3.18)-(3.23)), then outputs $\left(\omega_{i}{ }^{M}, \omega_{j}{ }^{M}\right)$, finally, adopts Eq.(3.6) to achieve $b_{i j}^{M}$.

\subsection{Deriving the upper and lower value of the adjustable matrix}

This section focuses on obtaining the value of $b_{i j}^{L \prime}$ and $b_{i j}^{U}$. The modified matrix is a combination of the adjustable matrix and original matrix based on definition 2; then the adjustable matrix should have the minimum fuzziness and maximum preservation of the original matrix's pattern. If $B^{\prime}$ is minimum fuzziness, then fuzziness of B will mostly come from $\mathrm{A}$, and $\mathrm{B}$ will be more similar with $\mathrm{A}$. In fact, the minimum fuzziness of $\mathrm{B}^{\prime}$ could reduce uncertainty factors of $\mathrm{B}$. If $\mathrm{B}^{\prime}$ could maximally preserve the pattern of $\mathrm{A}$, then the combination of $\mathrm{B}^{\prime}$ and $\mathrm{A}$ could reach to the most potential of similarity with $A$. We propose theorem 1 to obtain the value of $b_{i j}^{L \prime}$ and $b_{i j}^{U \prime}$ based on the above theory.

Theorem 1. The optimal solution vector is: $\omega_{i}^{M}$, then $\left(B_{i j}^{M}\right)^{\prime}=\frac{\omega_{i}{ }^{M}}{\omega_{j}{ }^{M}}, \mathrm{i}=1,2, \ldots, \mathrm{n}$. Set $\mathrm{C}_{\mathrm{L}}, \mathrm{C}_{\mathrm{U}}$ are arbitrary positive constants. Define the value of $\omega_{i}{ }^{L}, \omega_{i}^{U}$ in the following formulas:

$$
\omega_{i}^{L}=C_{L} . \omega_{i}^{M}
$$

Where $C_{L}=\min \left\{\frac{\sum_{j=1}^{n} a_{i j}^{M}}{\sum_{j=1}^{n} a_{i j}^{L}}, \frac{\left(\prod_{j=1}^{n} a_{i j}^{M}\right)^{1 / n}}{\left(\prod_{j=1}^{n} a_{i j}^{L}\right)^{1 / n}}\right\}$

and $\omega_{i}^{U}=C_{U} \cdot \omega_{i}^{M}$ 
Where $C_{U}=\max \left\{\frac{\sum_{j=1}^{n} a_{i j}^{M}}{\sum_{j=1}^{n} a_{i j}^{U}}, \frac{\left(\prod_{j=1}^{n} a_{i j}^{M}\right)^{1 / n}}{\left(\prod_{j=1}^{n} a_{i j}^{U}\right)^{1 / n}}\right\}$

Then the value of $b_{i j}^{L \prime}$ and $b_{i j}^{U \prime}$ is defined as:

$$
b_{i j}^{L \prime}=\omega_{i}^{L} / \omega_{j}^{L} \text { and } b_{i j}^{U \prime}=\omega_{i}^{U} / \omega_{j}^{U}
$$

Proof. The value of $\mathrm{C}_{\mathrm{L}}$, and $\mathrm{C}_{U}$ should satisfy two conditions: one is minimum fuzziness of $\omega_{i}^{M}$, and the other one is maximally maintaining similarity of original matrix $\tilde{A}$.

By the first condition, we can get:

$$
\begin{aligned}
& \omega_{i}^{L} \leq \omega_{i}^{M} \leq \omega_{i}^{U}, \\
& \omega_{i}^{L}=C_{\min } \omega_{i}^{M}, \text { and } \omega_{i}^{U}=C_{\max } \omega_{i}^{M}
\end{aligned}
$$

By the second condition, we need to consider the distance between $a_{i j}^{L}, a_{i j}^{M}, a_{i j}^{U}$, maintain the relationship among the original matrix, and make the new matrix closest to the original matrix's pattern. It is a way to find out the smallest coefficient between $a_{i j}^{L}$ and $a_{i j}^{M}$, and the smallest coefficient between $a_{i j}^{M}$ and $a_{i j}^{U}$. We can get the following formulas based on the second condition:

$$
\begin{gathered}
C_{\min }=\min \left\{\frac{\sum_{j=1}^{n} a_{i j}^{M}}{\sum_{j=1}^{n} a_{i j}^{L}}, \frac{\left(\prod_{j=1}^{n} a_{i j}^{M}\right)^{1 / n}}{\left(\prod_{j=1}^{n} a_{i j}^{L}\right)^{1 / n}}\right\} \\
C_{\max }=\max \left\{\frac{\sum_{j=1}^{n} a_{i j}^{M}}{\sum_{j=1}^{n} a_{i j}^{U}}, \frac{\left(\prod_{j=1}^{n} a_{i j}^{M}\right)^{1 / n}}{\left(\prod_{j=1}^{n} a_{i j}^{U}\right)^{1 / n}}\right\}
\end{gathered}
$$

Therefore, it has been proven that formula (3.25) and (3.26) always exist and they can be corrected by (3.27) and (3.28).

\subsection{An Algorithm to obtain Modified PCM}

The adjustable matrix is derived by combining section 3.1 and 3.2. The expected modified PCM can be gotten based on definition 2. A proposed algorithm is to conclude the steps of how to obtain the modified consistency PCM.

\section{Algorithm 1: Find modified PCM}

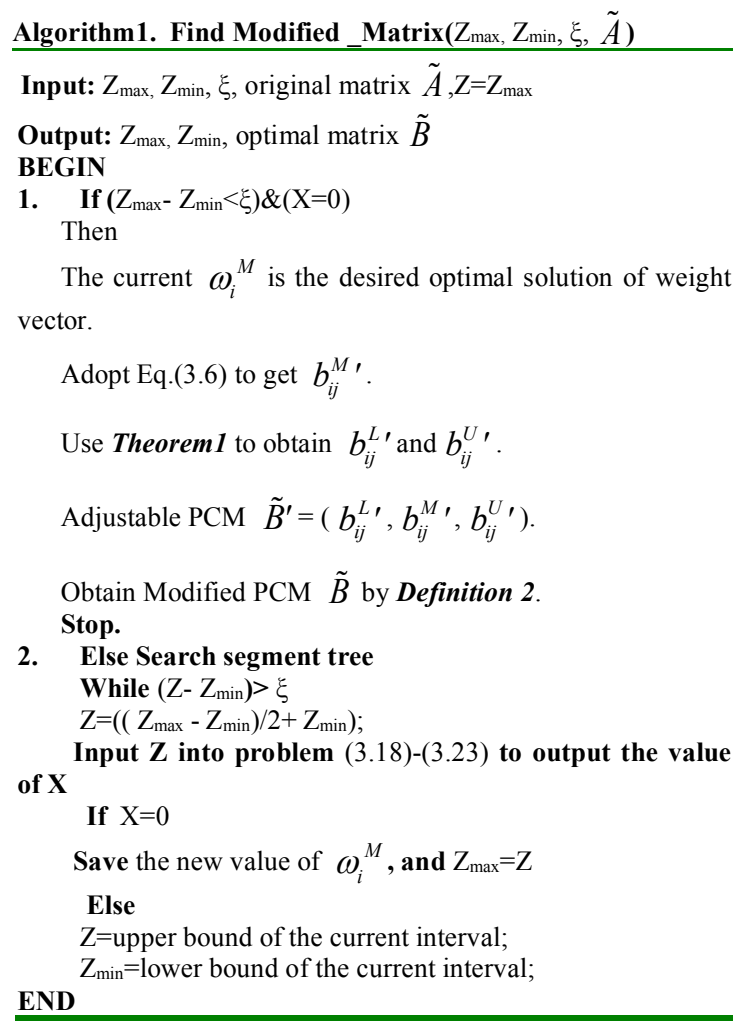

\section{Numerical illustration and comparison with crisp numbers}

\subsection{First illustration}

We run the experiments by software Matlab (R2009a) on a personal computer with $\operatorname{Intel}(\mathrm{R})$ Core(TM) 2.2 GHZ and 4 G RAM. First we test crisp numbers by using algorithm 1 , and then compare with references. Consider the following inconsistency PCM with crisp numbers in [33]:

$$
\mathrm{A}=\left(\begin{array}{l}
143134 ; \frac{1}{4} 173 \frac{1}{5} 1 ; \\
\frac{1}{3} \frac{1}{7} 1 \frac{1}{5} \frac{1}{5} \frac{1}{6} ; 1 \frac{1}{3} 511 \frac{1}{3} \\
\frac{1}{3} 551113 ; \frac{1}{4} 163 \frac{1}{3} 1
\end{array}\right)
$$

For PCM A, $\lambda_{\text {max }}=7.4199, \mathrm{CR}=0.2151>0.1$, the principal eigenvector is: $\omega=(0.32080 .13950 .0348$ $0.12850 .23740 .1391)^{\mathrm{T}}$.

The value of $\mathrm{CR}$ is much more than 0.1 . Therefore, we will adopt algorithm 1 to obtain the new consistency PCM.

Demonstration of Algorithm 1: 
Input the initial value of $\tilde{A}$, initial value of $\xi, Z_{\max }$, and $Z_{\min }$

1). The original matrix is $\tilde{\mathrm{A}}=\left(a_{i j}^{L}, a_{i j}^{M}, a_{i j}^{U}\right)$, as shown here, crisp number is a specific case of a fuzzy number in our model. Set $a_{i j}^{L}=a_{i j}^{M}=a_{i j}^{U}=A$.

$2)$. The acceptable precise degree is $\xi=0.1$. Here, we adopt 0.1 as an example.

3). The initial value of $Z_{\max }=5.7655$ which is obtained by Eq.(3.7), $Z_{\min }=0$, and the initial $Z=5.7655$. Algorithm 1 has a solution for the first $Z$ value. To better demonstrate, we start from the second iteration.

\section{Begin second time:}

Step1. $\left(Z_{\max }-Z_{\min }\right)>\xi$; then go to 2 ;

Step2. Search segment tree

$$
\left(Z_{\max }-Z_{\min }\right)>\xi \text {, then }
$$

Set new $Z=\left(\left(Z_{\max }-Z_{\min }\right) / 2+Z_{\min }\right)=2.8828$;

Input $Z$ into problem (3.18)-(3.23) to output the value of $X=1$,

$\mathrm{Z}=5.7655 ; \mathrm{Z}_{\min }=2.8828$; Go to step 1 .

\section{Remark 2.}

1). The value of $\xi$ is a stopping sign. If current value of $\xi$ is less than 0.1 , stop the calculation process.

2). The value of $X$ can determine how to change current $Z$ value. If $X=0$, it means there is a solution for problem (3.18)-(3.23), then save the value of vector $\omega$ and set $Z_{\max }=Z$; else $X=1$, it means there is not a solution for problem (3.18)-(3.23), then set $Z_{\min }=$ lower bound of the current interval, $Z=$ upper bound of the current interval.

$3)$. It needs a seven-time iteration to find out the final optimum vector of inconsistency PCM A. The detailed information of each parameter is shown in Table 1. Let $\omega_{\mathrm{i}}$ be the obtained vector of each feasible solution. $\omega_{5}$ is the optimum vector for the new consistency PCM B when $\beta=0.4$ in definition 2 .

The new obtained consistency PCM B is:

$$
\mathrm{B}=\left(\begin{array}{llllll}
1.0000 & 2.2560 & 4.5757 & 1.9544 & 1.3079 & 2.6896 \\
0.4433 & 1.0000 & 4.9561 & 2.3408 & 0.3417 & 1.1922 \\
0.2185 & 0.2018 & 1.0000 & 0.3482 & 0.1501 & 0.2559 \\
0.5117 & 0.4272 & 2.8720 & 1.0000 & 0.4312 & 0.5093 \\
0.7646 & 2.9267 & 6.6602 & 2.3190 & 1.0000 & 2.8444 \\
0.3718 & 0.8388 & 3.9085 & 1.9634 & 0.3516 & 1.0000
\end{array}\right)
$$

Table 1

Parameter values for each iterative time

\begin{tabular}{|l|l|l|l|l|l|l|}
\hline $\begin{array}{l}\text { Itera- } \\
\text { tive } \\
\text { time }\end{array}$ & $Z_{\max }$ & $Z_{\min }$ & $Z$ & $\xi$ & $X$ & $\omega$ \\
\hline 1 & 5.7655 & 0 & 5.7655 & 5.7655 & 0 & $\omega 1$ \\
\hline
\end{tabular}

\begin{tabular}{|l|l|l|l|l|l|l|}
\hline 2 & 5.7655 & 2.8828 & 2.8828 & 2.8828 & 1 & - \\
\hline 3 & 5.7655 & 2.8828 & 4.3242 & 1.4414 & 0 & $\omega 2$ \\
\hline 4 & 4.3242 & 2.8828 & 3.6035 & 0.7207 & 0 & $\omega 3$ \\
\hline 5 & 3.6035 & 2.8828 & 3.2431 & 0.3603 & 0 & $\omega 4$ \\
\hline 6 & 3.2431 & 2.8828 & 3.0629 & 0.1801 & 0 & $\omega 5$ \\
\hline 7 & 3.0629 & 2.9729 & 3.0629 & 0.0787 & 1 & - \\
\hline$\omega 5$ & $(1.0000,0.6494,0.1649,0.3273,1.3299,0.4844)$ \\
\hline
\end{tabular}

Three consistencies of PCM B are obtained under different precise degrees. To evaluate the effective ness of obtained PCM B, CR (consistency index), $\delta$ (Eq.2.5), $\sigma$ (Eq.2.6), iteration times (mark "II" in Table 2), and COP (Condition of Order Preservation) are shown in Table 2.

The consistency value of the original A is 0.2151 , whereas the consistency of new obtained PCM B is around 0.03 . The effective parameters $\delta$ (less than 2) and $\sigma$ (less than 1) are both at an acceptable level. The aim to calculate COP is to make sure the obtained PCM could preserve of the order of preference and preserve the order of intensity of preference of original PCM A. COP tests every pair of weights and the proportion among every pairs' weights. The iteration times are less than 14.

Table 2

Effective analysis for algorithm 1 based on five parameters

\begin{tabular}{|l|l|l|l|l|c|}
\hline PCM & CR & $\delta$ & $\sigma$ & COP & II \\
\hline $\mathrm{A}$ & 0.2151 & 0 & 0 & & - \\
\hline $\mathrm{B}(\xi=0.1)$ & 0.0325 & 1.6602 & 0.9916 & Keep & 7 \\
\hline $\mathrm{B}(\xi=0.01)$ & 0.0325 & 1.6294 & 0.9868 & Keep & 11 \\
\hline $\mathrm{B}(\xi=0.001)$ & 0.0324 & 1.6291 & 1.0308 & Keep & 14 \\
\hline
\end{tabular}

\subsection{Comparison with References}

$\mathrm{Xu}$ and Wei [4] defined the original matrix A (the elements are $\mathrm{a}_{\mathrm{ij}}$ ) that can be replaced by the new matrix B (the elements are $b_{i j}$ ), which is showed in following equation:

$$
b_{i j}=a_{i j}^{\alpha}\left(\omega_{i} / \omega_{j}\right)^{1-\alpha}
$$

Where $\alpha$ is the positive value which is less than, but approaching to 1 .

Cao et al. [5] proposed a equation to obtain the new matrix $B$, which is showed the following:

$$
b_{i j}=\left(\omega_{i} / \omega_{j}\right) \circ D^{\prime}
$$

Where ois the symbol of Hadamard product; for example, $\mathrm{A}=\mathrm{BoC}$ means $a_{i j}=b_{i j} \times c_{i j}, \forall i, j=1, \cdots, n$, and $D^{\prime}$ is the modified deviation matrix, which is showed in the following equation:

$$
D^{\prime}=\left[d_{i j}^{\prime}\right]=\gamma D \oplus(1-\gamma) D I
$$


Where $\mathrm{D}$ is the deviation matrix, DI is a zero deviation matrix when $\left[d_{i j}\right]=1$. The value of $\gamma$ is between 0 and 1 .

$\alpha$ and $\gamma$ has different meaning for two papers; but, the two parameters should be as close to 1 as possible. In two papers, they mentioned that $\alpha=\gamma=0.98$ is the most suitable value to get the optimal new matrix. We compare with two references in two situations: one is the required Critical Ratio (CR) less than 0.1 (Table 3); the other one is the required Critical Ratio (CR) close to 0 (Table 3 ).

Algorithm 1 can reach a CR value lower than reference [4] and [5] when CR is approaching to 0.1, and at the same time, the method could achieve lower values of $\delta$ and $\sigma$ in short iterative times. We also rank the priority weight $\omega$ derived from A. The ranking results are same in two similar weights, which mean the priority weight $\omega$ which derived from our method is acceptable. The iterative times for reference [3] and [4] are quite large, 820 and 1619 when
$\mathrm{CR}$ is approaching to 0 , which will cost the running time. The value of $\delta$ and $\sigma$ in these two references is far away from the acceptable value, which means the priority weight is not acceptable at all; But, the iterative times of our method is less than 10 times, the value of $\delta$ is less than 4 , and the value of $\sigma$ is less than 2, which means our result is acceptable to some extent. On the basis of these results, we go to discover the difference of COP parameter in our method and two references. Cao et al. is closer to the original matrix's vector, but the gap among $\mathrm{Cao}, \mathrm{Xu}$, et al. and our method is very narrow (less than 1). The three methods have almost the same effectiveness in terms of the COP parameter. Thus, our method is also acceptable in this parameter.

In conclusion, Algorithm 1 can preserve more original information and obtain more consistent new matrix in short iterative times for PCMs with crisp elements.

Table 3

Effective analysis of Algorithm 1 by comparing with references based on parameters

\begin{tabular}{|l|c|l|l|l|l|l|l|l|l|}
\hline \multicolumn{1}{|c|}{ CR $\leq 0.1$} & \multicolumn{3}{c|}{ CR=0 } \\
\hline \multicolumn{1}{|c|}{ Methods } & $\begin{array}{l}\text { Iteration } \\
\text { Time }\end{array}$ & CR & $\delta$ & $\sigma$ & $\lambda_{\max }$ & Methods & $\begin{array}{l}\text { Iteration } \\
\text { Time }\end{array}$ & $\delta$ & $\sigma$ \\
\hline Xu and Wei [4] & 12 & 0.0972 & 1.845 & 0.589 & 8.955 & Xu and Wei $[4]$ & 820 & 10.4648 & 1.9069 \\
\hline Cao et al.[5] & 18 & 0.0997 & 1.713 & 0.448 & 8.9844 & Cao et al.[5] & 1619 & 11.0308 & 2.0714 \\
\hline $\begin{array}{l}\text { Our method( } \xi=0.05, \\
\beta=0.8)\end{array}$ & 8 & 0.0964 & 1.1572 & 0.4710 & 8.9017 & $\begin{array}{l}\text { Our meth- } \\
\text { od }(\xi=0.05, \beta=0.2)\end{array}$ & 8 & 3.2554 & 1.4458 \\
\hline $\begin{array}{l}\text { Our method( } \xi=0.1, \\
\beta=0.8)\end{array}$ & 7 & 0.0964 & 0.7354 & 0.4689 & 8.9017 & $\begin{array}{l}\text { Our meth- } \\
\text { od }(\xi=0.1, \beta=0.2)\end{array}$ & 7 & 3.5117 & 1.5088 \\
\hline $\begin{array}{l}\text { Our } \operatorname{method}(\xi=0.2, \\
\beta=0.8)\end{array}$ & 6 & 0.0964 & 1.2273 & 0.4993 & 8.9017 & $\begin{array}{l}\text { Our meth- } \\
\text { od }(\xi=0.2, \beta=0.2)\end{array}$ & 6 & 3.6985 & 1.7867 \\
\hline
\end{tabular}

\section{Numerical illustration and comparison with fuzzy numbers}

\subsection{First analysis for pairwise comparison matrix with fuzzy elements}

To judge the effectiveness of Algorithm 1 for PCMs with fuzzy elements, we analyze several parameters including the inconsistency index NI [19], consistency index CCI $[28,29]$, $\delta_{\text {fuzzy }}, \sigma_{\text {fuzzy }}, \mathrm{COP}$, iteration times and running time. There is no evidence showing the thresholds of NI. The thresholds for CCI are provided as: $\mathrm{CCI}=0.3147$ for $\mathrm{n}=3$, $\mathrm{CCI}=0.3526$ for $\mathrm{n}=4$ and $\mathrm{CCI}=0.370$ for $\mathrm{n}>4$ based on Aguaron's [26]. Considering the following PCM with fuzzy elements:

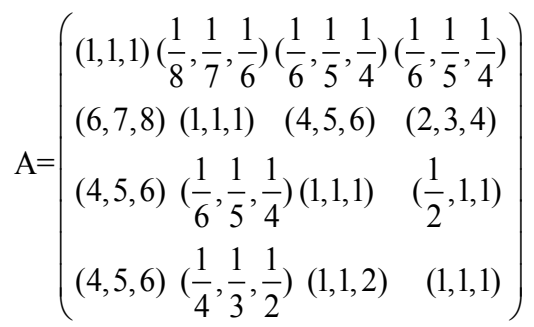

For matrix $\mathrm{A}, \mathrm{NI}(\mathrm{A})=0.6704, \mathrm{CCI}=0.3746$. It is an inconsistent PCM with fuzzy elements. We adopt Algorithm 1 to obtain the modified new matrix under different parameters. The results are shown in Table 4. In Table 4, the inconsistency index NI has been reduced by 0.1 and the consistency index CCI, is around 0.1 , which is much less than 0.3147 and indicates the modified matrix can reach to consistent requirement; $\delta_{\text {fuzzy }}$ satisfies $0<\delta_{\text {fuzzy }}<2$ and $\sigma_{\text {fuzzy }}$ satis- 
fies $0<\sigma_{\text {fuzzy }}<1$, which shows the obtained new matrix is within acceptable distance with $\mathrm{A}$; the value of COP which is gotten from priority vector $\omega$ of $B$ can preserve order of preference and order of intensity preference, which present Bcan maintain the pat- tern of A (similarity). Meanwhile, algorithm 1 has high convergence speed based on the less iteration times and running time. The gap among these four matrixes is quite narrow, $\mathrm{B}(\xi=0.1, \beta=0.5)$ is the best choose when we select the smallest consistency index. Table 4.

Effective Analysis for algorithm 1 based on nine parameters

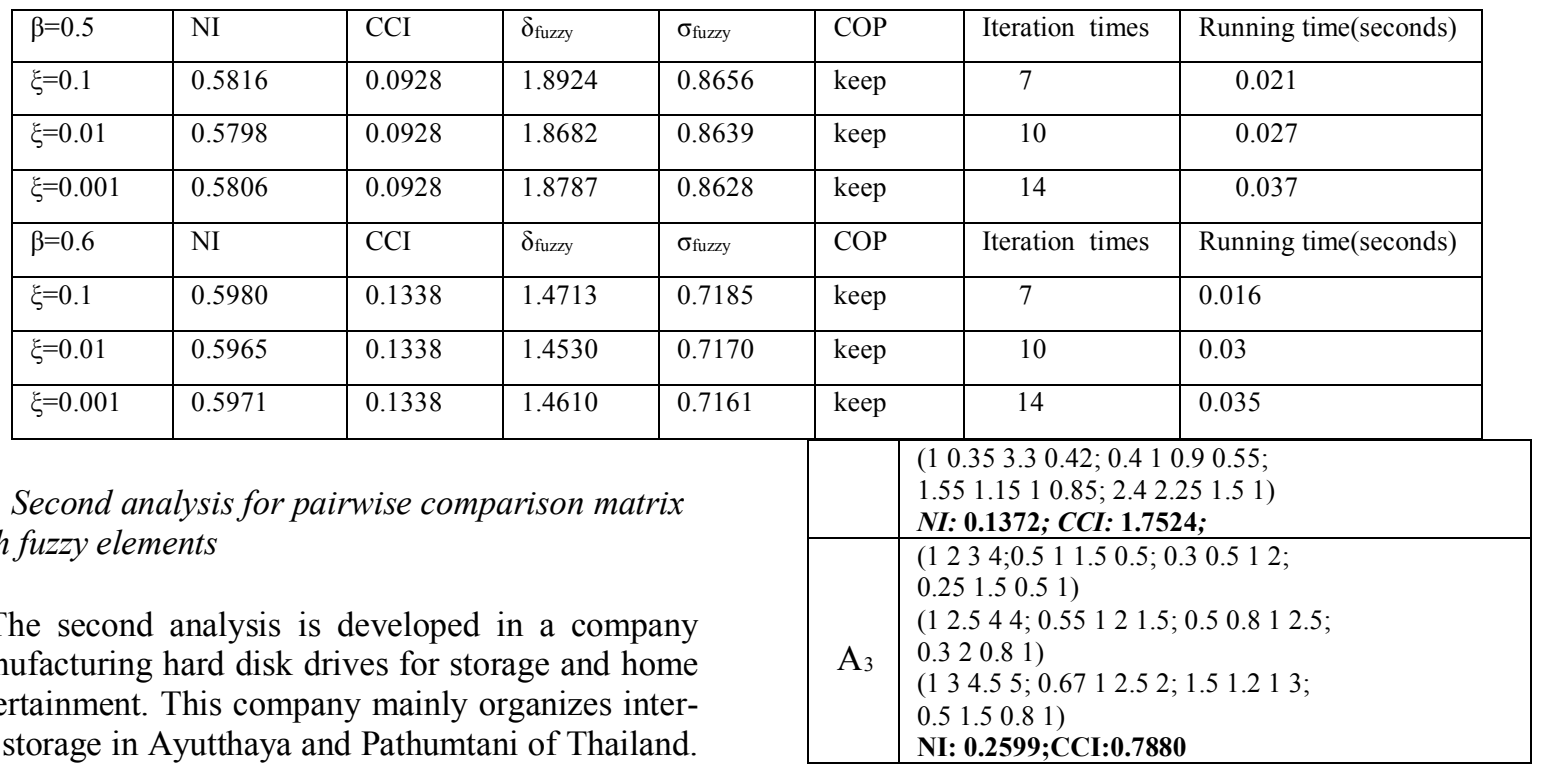
To better handle sustainability of a plant in Pathumtani, we define three main criteria: economic, environmental, social. Then define different sub-criteria for these three main criteria. To make a believable decision of which criteria play fundamental roles in sustainability, decision makers give the fuzzy PCMs. Algorithm 1 analyzes these PCMs and provide the closest consistency PCMs for inconsistency ones. Twenty-eight fuzzy matrixes are inconsistency matrixes, and three of them are selected in Table 4.

$$
\text { Table } 4
$$

Inconsistency PCMs with fuzzy elements

\begin{tabular}{|c|c|}
\hline$A_{1}$ & 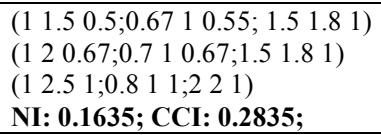 \\
\hline $\mathrm{A}_{2}$ & 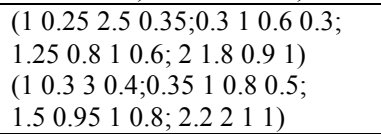 \\
\hline
\end{tabular}

Algorithm 1 is adopted to obtain the modified PCMs. Table 5 displays the results. The results show the new modified PCMs have good consistency level because of all NI value less than 0.1 and all CCI value less than 0.3147. Meanwhile, the new modified PCMs are closest to original PCMs because $\delta_{\text {fuzzy }}$ and $\delta_{\text {fuzzy }}$ are in an acceptable range, and the COP value shows that the new modified PCMs preserve order of preference and order of intensity preference. Ten PCMs are studied in Figure 1. The results show that Algorithm 1 could effectively reduce the inconsistency of original PCM (reduced the value of NI and $\mathrm{CCI}$ ) and preserve the information of original PCM. Algorithm 1 can also approach to its limits faster than [45]. The maximum iteration times for Algorithm 1 is less than twenty, even when $\xi=0.0001$, while the maximum iteration times for [5] and [4] are 1619 and 820 respectively. The convergence rate of Algorithm 1 has low growth rate. Figure 2 shows the results.

Modified PCMs with fuzzy elements

$\mathrm{B}_{1} \quad(1.00001 .37840 .8130 ; 0.72661 .00000 .6855 ; 1.12821 .45451 .0000)$

(1.0000 1.4460 0.8376; $0.76501 .00000 .7132 ; 1.19571 .48331 .0000)$

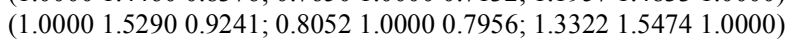

NI: $0.0895 ;$ CCI: $0.0235 ; \delta_{\text {fuzzy }}: 0.3130 ; \delta_{\text {fuzzy }}: 0.4270$ 


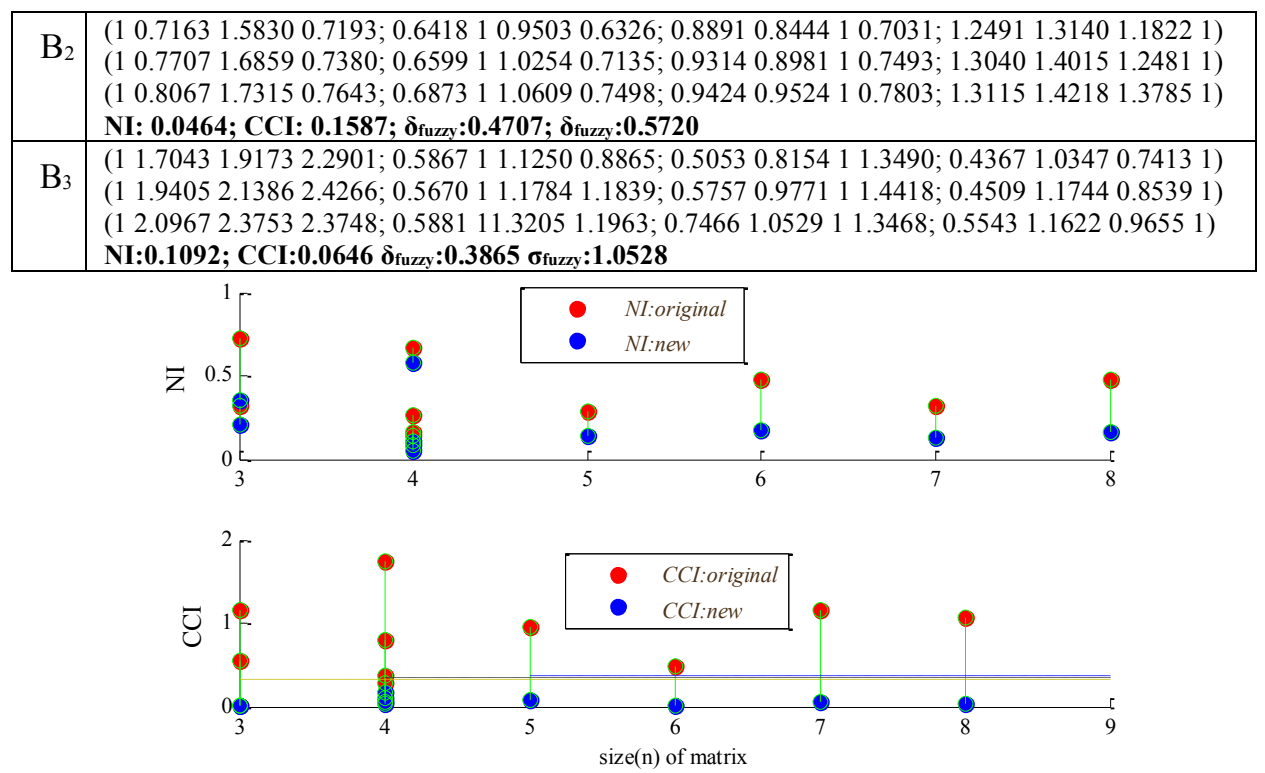

Fig. 1. Consistency analysis for obtained new PCMs with fuzzy elements
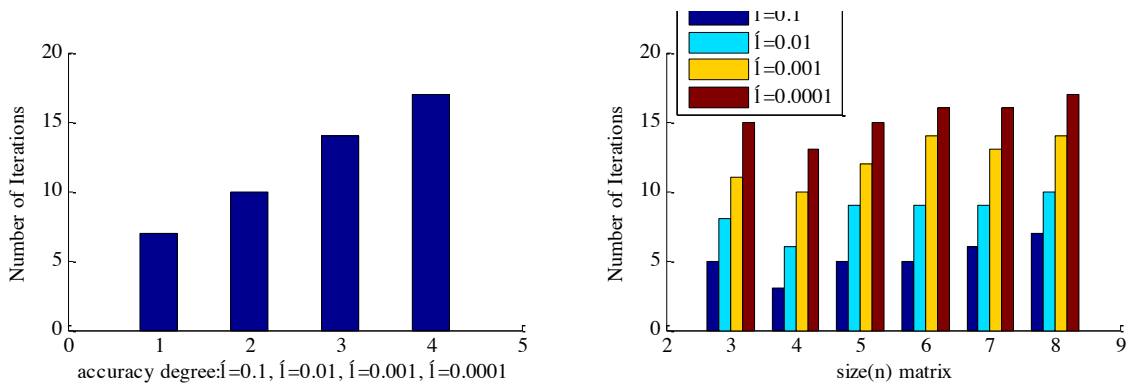

Fig. 2. Convergence rate for Algorithm 1

\section{Conclusions}

Algorithm 1 has been proposed to derive a consistent PCM with crisp or fuzzy elements from an inconsistent one. The presented approach is tested in several examples. The experiments results in Table 3 conclude the effectiveness of Algorithm 1 by comparing with Cao et al. [5] and $\mathrm{Xu}$ and Wei [4] based on PCMs with crisp elements. Algorithm 1 can retain more original information and achieve lower value of $\delta$ and $\sigma$ when both $\alpha$ [4] and $\gamma$ [5] approach to 1. Tables 4 and 5 summarize the effectiveness of Algorithm 1 for PCMs with fuzzy elements. The modified PCMs with fuzzy elements can maintain original information and reach to acceptable consistency level as well.
Two effective criteria $\delta$ and $\sigma$ show how the distance between modified PCM and original PCM is acceptable for both crisp and fuzzy elements. A new effective criterion $\mathrm{COP}$ reflects that the modified PCM resembles the original PCM (crisp and fuzzy elements). In conclusion, this approach could enhance the quality of vague and inaccurate data for decision makers, and also can better handle the inconsistency problems of AHP and Fuzzy AHP.

However, $\beta$ in Algorithm 1 has a different meaning with $\alpha$ and $\gamma$. The true meaning of $\beta$ is the proportion of the original matrix to an adjustable matrix. The approximate prefect range of $\beta$ is $[0.25,0.8]$ based on experiments' results, and there is no clue to which value is the best one for $\beta$ as the inconsistency level of PCMs are differs. In future research, it would be interesting to figure out the exact prefect range of $\beta$. Meanwhile, efforts should be made to test Algorithm 1 for more cases. 


\section{References}

[1] T.L. Saaty, 'The Analytic Hierarchy Process', McGraw-Hill, New York, 1980.

[2] Buckley, J.J., 'Fuzzy Hierarchical Analysis', Fuzzy Sets and Systems, 17 (1985), 233-247.

[3] S. Karapetrovic, E.S. Rosenbloom, A quality control approach to consistency paradoxes in AHP, Eur. J. Oper. Res. 119 (1999) 704-718.

[4] Z.S. Xu, C.P. Wei, A consistency improving method in the analytic hierarchy process, Eur. J. Oper. Res. 116 (1999) 443449.

[5] Cao, D., L.C. Leung, and J.S. Law, 'Modifying Inconsistent Comparison Matrix in Analytic Hierarchy Process: A Heuristic Approach', Decision Support Systems, 44 (2008), 944-953.

[6] $\mathrm{Xu}$, Yejun, and Huimin Wang, 'Eigenvector Method, Consistency Test and Inconsistency Repairing for an Incomplete Fuzzy Preference Relation', Applied Mathematical Modelling, 37 (2013), 5171-5183.

[7] Pérez, I.J., Cabrerizo, F.J., Herrera-Viedma, E., 2010. A mobile decision support system for dynamic group decisionmaking problems. Systems, Man and Cybernetics, Part A: Systems and Humans, IEEE Transactions on 40, 1244-1256.

[8] Lee, A.H.I., Chen, W.-C., Chang, C.-J., 2008. A fuzzy AHP and BSC approach for evaluating performance of IT department in the manufacturing industry in Taiwan. Expert Systems with Applications 34, 96-107.

[9] G. Kou, D. Ergu, Y. Peng, and Y. Shi, "A New Consistency Test Index for the Data in the AHP/ANP," in Data Processing for the AHP/ANP, Springer, 2013, pp. 11-27.

[10]HQ. Zhang, Y. Ouzrout, A. Bouras, V. Della Selva, M. Savino. "Selection of optimal Product Lifecycle Management components based on AHP Methodologies." International Conference on Advanced Logistics and Transport (ICALT'2013). Sousse, Tunisia, 29-31May, pp. 523-528, 2013.

[11]HQ. Zhang, Y. Ouzrout, A. Bouras, A. Mazza, M. Savino . "PLM Components Selection Based on a Maturity Assessment and AHP Methodology". International Conference on Product Lifecycle Management Conference (PLM'13), Nantes, France, 6-10July, 2013.

[12]Torfi, Fatemeh, Reza Zanjirani Farahani, and Shabnam Rezapour. "Fuzzy AHP to determine the relative weights of evaluation criteria and Fuzzy TOPSIS to rank the alternatives." Applied Soft Computing 10.2 (2010): 520-528.

[13]Yang, M., Khan, F. I., \& Sadiq, R. Prioritization of environmental issues in offshore oil and gas operations: A hybrid approach using fuzzy inference system and fuzzy analytic hierarchy process. Process Safety and Environmental Protection, 89(1), 22-34. (2011)

[14]Aguar'on, J., Moreno-Jim 'enez, J.M., The geometric consistency index: Approximated threshold, European Journal of Operational Research, 147, 137-145 (2003)

[15]S.I. Gass, T. Rapcsák, Singular value decomposition in AHP European Journal of Operational Research 154 (2004) 573584 ISSN 0377-2217.

[16]W.E. Stein, P.J. Mizzi, The harmonic consistency index for the analytic hierarchy process, European Journal of Operational Research 177(2007) 488-497 ISSN 0377-2217.

[17]T.L. Saaty, Multi-criteria Decision Making-The Analytical Hierarchy Process, Vol. I, RWS Publications, Pittsburgh, 1991 ISBN0-9620317-2-0.

[18]L.C. Leung, D. Cao, On consistency and ranking of alternatives in fuzzy AHP, European Journal of Operational Research 124 (2000) 102-113 ISSN 0377-2217.
[19]Ramík, Jaroslav, and Petr Korviny. "Inconsistency of Pairwise Comparison Matrix with Fuzzy Elements Based on Geometric Mean." Fuzzy Sets and Systems 161 (11) (June 1): 1604-1613. (2010)

[20]Dubois, D. The role of fuzzy sets in decision sciences: Old techniques and new directions. Fuzzy Sets and Systems, 184(1), 3-28. (2011).

[21]Brunelli, M. A note on the article "Inconsistency of pair-wise comparison matrix with fuzzy elements based on geometric mean" [Fuzzy Sets and Systems 161 (2010) 1604 1613]. Fuzzy Sets and Systems, 176(1), 76-78.

[22] Saaty, T.L., Hu, G., Ranking by the eigenvector versus other methods in the analytic hierarchy process. Applied Mathematical Letters 11 (4), 121-125. 1998.

[23] Saaty, T.L., Decision-making with the AHP: Why is the principal eigenvector necessary. European Journal of Operational Research 145 (1), 85-91. 2003

[24]Bana e Costa, Carlos A., and Jean-Claude Vansnick. 2008. “A Critical Analysis of the Eigenvalue Method Used to Derive Priorities in AHP." European Journal of Operational Research 187 (3) (June 16): 1422-1428.

[25]Mahmoud zadeh, Morteza, and Ali R. Bafandeh. "A New Method for Consistency Test in Fuzzy AHP." Journal of Intelligent and Fuzzy Systems 25 (2): 457-461. 2013.

[26]J. Aguarón, J.M.Moreno-Jiménez, The geometric consistency index: Approximated thresholds, European Journal of Operational Research 147 (2003) 137-145.

[27]E. Bulut, O. Duru, T. Keçeci, S. Yoshida, Use of consistency index, decision prioritization and direct numerical inputs for generic Fuzzy-AHP modeling: A process model for shipping asset management, Expert Systems with Applications 39 (2012) 1911-1923.

[28]Dopazo, E., K. Lui, S. Chouinard, and J. Guisse. "A Parametric Model for Determining Consensus Priority Vectors from Fuzzy Comparison Matrices." Fuzzy Sets and Systems (August). 2013.

[29]P. De Jong, A statistical approach to Saaty's scaling methods for priorities, Journal of Mathematical Psychology 28 (1984) 467-478.

[30]T. S. Almulhim, L. Mikhailov, and D.-L. Xu, "Deriving Weights from Group Fuzzy Pairwise Comparison Judgement Matrices," in Advances in Information Systems and Technologies, Springer, 2013, pp. 545-555.

[31]Zimmermann, H.J., 2001. Fuzzy set theory-and its applications. Springer.

[32] Winston, Wayne L., Munirpallam Venkataramanan, and Jeffrey B. Goldberg. Introduction to mathematical programming. Vol. 1. Thomson/Brooks/Cole, 2003.

[33] Saaty TL. Fundamentals of decision making and priority theory with the analytic hierarchy process. Pittsburgh: RWS Publications; 2000.

[34]Deng, H., 1999. Multicriteria analysis with fuzzy pairwise comparison, in: Fuzzy Systems Conference Proceedings, 1999. FUZZ-IEEE '99. 1999 IEEE International. Presented at the Fuzzy Systems Conference Proceedings, 1999. FUZZIEEE '99. 1999 IEEE International, pp. 726-731 vol.2.

[35]Chiclana, F., Herrera-Viedma, E., Alonso, S., Herrera, F., 2009. Cardinal Consistency of Reciprocal Preference Relations: A Characterization of Multiplicative Transitivity. IEEE Transactions on Fuzzy Systems 17, 14-23.

[36]Cabrerizo, F.J., Pérez, I.J., Herrera-Viedma, E., 2010. Managing the consensus in group decision making in an unbalanced fuzzy linguistic context with incomplete information. Knowledge-Based Systems 23, 169-181. 